\title{
CURVAS REGULARES E EQUAÇÕES DE FRENET
}

Thiago Mariano Viana ${ }^{1}$, Dr. Fernando Pereira Souza ${ }^{2}$

${ }^{1}$ Aluno do curso de Matemática - CPTL/UFMS, bolsista do grupo PET Matemática CPTL/UFMS; ${ }^{2}$ Professor do curso de Matemática - CPTL/UFMS. E-mail: tmviana2000@gmail.com

\section{RESUMO}

A geometria diferencial surge da união da geometria e o calculo diferencial como uma ciência aplicada, principalmente em questões originadas da cartografia, posteriormente passou a ser de grande utilidade na Astronomia e na Engenharia, e hoje é uma ferramenta muito útil para modelagem de problemas de várias áreas, como Biologia, Física. A grosso modo, geometria diferencial clássica é o estudo das propriedades locais das curvas e superfícies, e neste trabalho apresentamos um desenvolvimento introdutório da teoria local das curvas planas, visando mostrar alguns conceitos básicos importantes para compreensão do estudo do conteúdo de geometria que é essencial para o aluno que pretenda seguir um mestrado e/ou doutorado na área.

\section{INTRODUÇÃO E OBJETIVOS}

Esse trabalho aborda uma parte do estudo local da teoria das curvas. Vamos inicialmente trabalhar com curvas planas, pois muitos dos seus resultados podem ser apresentados de forma elementar. Elementar no sentido que os pré-requisitos necessários para compreensão do assunto são calculo I e geometria analítica. Entende-se por teoria local, como sendo o estudo do comportamento da curva na vizinhança de um de seus pontos.

Primeiro iremos apresentar os conceitos e definições de curvas parametrizadas diferenciáveis, e sua reparametrização. Iremos analisar suas características tanto quanto vetor tangente, vetor normal. E em um segundo momento será introduzido o referencial de Frenet nas curvas planas, e as formulas de Frenet.

\section{METODOLOGIA}

A pesquisa é exploratória, pois trata-se de um estudo baseado no livro Introdução à Geometria Diferencial, de Keti Tenemblat e no livro Geometria Diferencial das Curvas e Superfícies, de Manfredo Perdigão do Carmo. O trabalho foi discutido semanalmente com o orientador nas apresentações de seminários 


\section{RESULTADOS}

Para compreensão do trabalho não é exigido muitos pré-requisitos, no entanto é preciso um conhecimento prévio de calculo vetorial no espaço euclidiano e calculo diferenciável no espaço euclidiano.

Ao longo do trabalho desenvolvemos a teoria local das curvas planas necessária para a compreensão das proposições estudadas. Nossa linha de pesquisa segue os trabalhos desenvolvidos em [1] e [2].

\section{Curvas Planas}

Em geometria diferencial uma curva é descrita dando-se as coordenadas de seus pontos como funções de uma variável independente. Assim uma maneira natural de definirmos uma curva, é como um subconjunto, de certa forma unidimensional do espaço $\mathbb{R}^{2}$, através de funções diferenciáveis. Diremos que uma função real é diferenciável (ou suave) se ela possui em todos os pontos, derivadas de todas as ordens (não é uma definição elegante, mas é suficiente para desenvolvimento do trabalho).

Definição: Uma curva diferenciável parametrizada é uma aplicação diferenciável $\alpha: I \rightarrow \mathbb{R}^{3}$ de um intervalo aberto $I=(a, b)$ da reta real $\mathbb{R}$ em $\mathbb{R}^{2}$.

A palavra diferenciável na definição significa que $\alpha$ é uma correspondência que leva cada $t \in I$ em um ponto $\alpha(t)=(x(t), y(t)) \in \mathbb{R}^{2}$, de tal modo que as funções reais $x(t)$ e $y(t)$ são diferenciáveis. Diremos que a variável $t$ é o parâmetro da curva.

Exemplos:

a) A aplicação

$$
\alpha(t)=\left(x_{0}+a t, y_{0}+b t\right), t \in \mathbb{R}
$$

onde $a^{2}+b^{2} \neq 0$, é uma curva parametrizada diferenciável cujo traços é uma linha reta que passa pelo ponto $\left(x_{0}, y_{0}\right)$, paralela ao vetor de coordenada $(a, b)$ (veja Figura 1$)$.

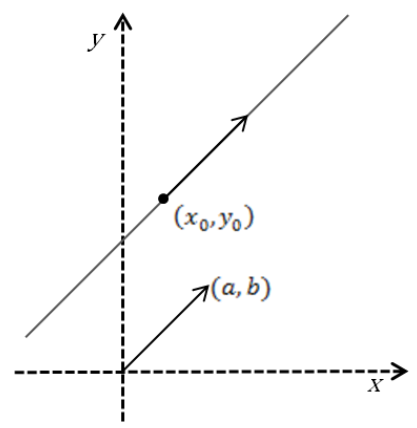

\section{Figura 1}


b) A aplicação $\alpha$, que para cada $t \in \mathbb{R}$ associa

$$
\alpha(t)=(\cos t, \operatorname{sen} t),
$$

é uma curva parametrizada diferenciável, cujo traço é uma circunferência de centro na origem e raio igual a 1.

Duas curvas parametrizadas diferenciáveis podem ter o mesmo traço. Por exemplo

$$
\begin{aligned}
& \alpha(t)=(t, 2 t), t \in \mathbb{R} \\
& \beta(r)=(2 r+1,4 r+2), r \in \mathbb{R}
\end{aligned}
$$

têm o traço da Figura 2

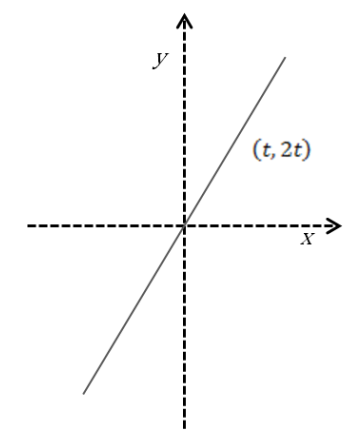

Figura 2

\section{Vetor Tangente e Curva Regular}

Denotando por $x^{\prime}(t)$ a derivada primeira ordem de $x(t)$ em um ponto $t$, e utilizamos notações análogas para as funções $y$ e $z$.

Definição: Seja $\alpha: I \rightarrow \mathbb{R}^{2}$ uma curva parametrizada diferenciável, que a cada $t \in \mathbb{R}$ associa $\alpha(t)=(x(t), y(t))$. O vetor

$$
\alpha^{\prime}(t)=\left(x^{\prime}(t), y^{\prime}(t)\right)
$$

é chamado vetor tangente (ou vetor velocidade) a $\alpha$ em $t$.

Exemplo:

a) Seja $\alpha: \mathbb{R} \rightarrow \mathbb{R}^{2}$ a curva parametrizada diferenciável que para cada $t \in \mathbb{R}$, associa

$$
\alpha(t)=(\cos t(2 \cos t-1), \operatorname{sen} t(2 \cos t-1)) .
$$

O vetor tangente a $\alpha$ em $t$ é igual a

$$
\alpha^{\prime}(t)=(\operatorname{sen} t-2 \operatorname{sen} 2 t, 2 \cos 2 t-\cos t) .
$$

Observamos que um vetor tangente á uma curva $\alpha$ é definido no parâmetro $t$, e não no ponto $\alpha(t)$, pois como visto no exemplo acima $\alpha\left(\frac{\pi}{3}\right)=\alpha\left(-\frac{\pi}{3}\right)=0$ (ver Figura 3) e, no entanto $\alpha^{\prime}\left(\frac{\pi}{3}\right) \neq \alpha^{\prime}\left(-\frac{\pi}{3}\right)$, portanto o vetor tangente ao traço da curva na origem de $\mathbb{R}^{2}$ não está bem definido. 


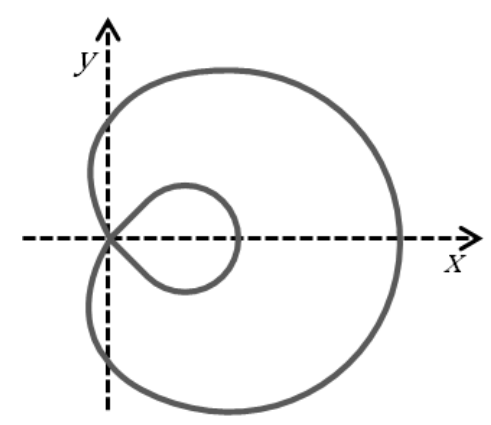

\section{Figura 3}

b) as duas curvas parametrizadas distintas

$$
\begin{aligned}
& \alpha(t)=(\cos t, \operatorname{sen} t), \\
& \beta(t)=(\cos 2 t, \operatorname{sen} 2 t),
\end{aligned}
$$

Onde $t \in(0-\epsilon, 2 \pi+\epsilon), \epsilon>0$, possuem o mesmo traço, o circulo $x^{2}+y^{2}=1$. No entanto obseve que o vetor tangente da segunda curva é o dobro da primeira (Figura 4).

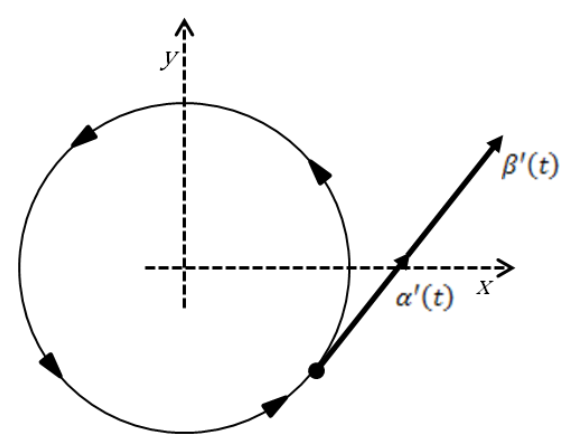

\section{Figura 4}

Seja $\alpha: I \rightarrow \mathbb{R}^{2}$ uma curva diferenciável parametrizada. Para cada $t \in I$ tal que $\alpha^{\prime}(t) \neq 0$, há uma reta bem definida contendo o ponto $\alpha(t)$ e o vetor $\alpha^{\prime}(t)$. Essa reta é chamada reta tangente a $\alpha$ em $t$. Para desenvolvimento da geometria diferencial das curvas é essencial a existência de uma reta tangente em todos os pontos. Portanto chamaremos de ponto singular de $\alpha$ um ponto $t \in I$ onde $\alpha^{\prime}(t)=0$, e restringirmos as nossas considerações às curvas sem pontos singulares.

Definição: Uma curva diferenciável parametrizada $\alpha: I \rightarrow \mathbb{R}^{2}$ é chamada regular se $\alpha^{\prime}(t) \neq 0$ para todo $t \in I$. 


\section{Mudança de Parâmetro; Comprimento de Arco}

Como vimos duas curvas podem ter o mesmo traço. Dada uma curva regular $\alpha$, podemos obter várias curvas regulares que tem o mesmo traço que $\alpha$. Da seguinte forma:

Seja $I$ e $J$ intervalos abertos de $\mathbb{R}, \alpha: I \rightarrow \mathbb{R}^{2}$ uma curva regular e $h: I \rightarrow J$ uma curva diferenciável $\left(C^{\infty}\right)$, cuja derivada de primeira ordem é não nula em todos os pontos de $J$ e tal que $h(J)=I$. Então a função composta

$$
\beta=\alpha \circ h: J \rightarrow \mathbb{R}^{2}
$$

é uma curva regular que tem o mesmo traço que $\alpha$, chamada de reparametrização de $\alpha$ por $h$. A função $h$ é dita mudança de parâmetro

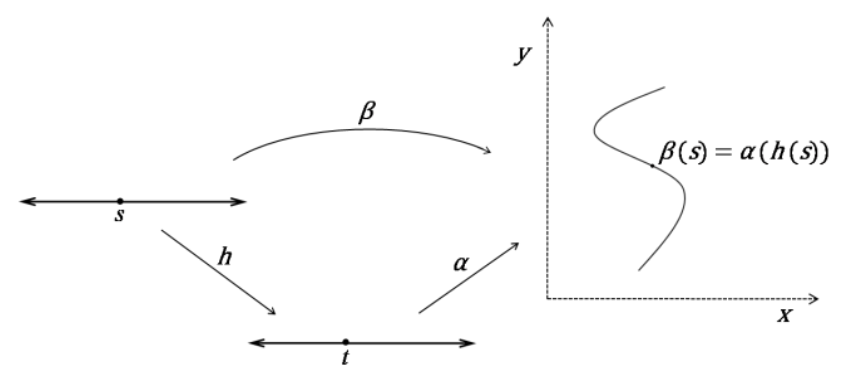

\section{Figura 5}

Seja $\alpha: I \rightarrow \mathbb{R}^{2}$ uma curva regular e fixemos $a$ e $b$ do intervalo $I$. Subdividindo o intervalo $[a, b]$ nos pontos $a=t_{0}<t_{1}<\cdots<t_{n}=b$, consideremos a soma $\sum_{i=1}^{n}\left|\alpha\left(t_{i}\right)-\alpha\left(t_{i-1}\right)\right|=l(\alpha, P)$, onde $P$ refere-se a partição dada. A norma $|P|$ da partição $P$ é definida por

$$
|P|=\max \left(t_{i}-t_{i-1}\right), i=1, \ldots, n \text {. }
$$

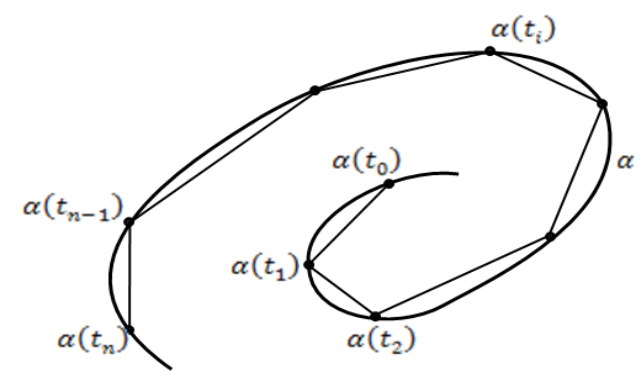

Figura 6

Geometricamente, $l(\alpha, P)$ é o comprimento de um polígono inscrito em $\alpha([a, b])$ com vértices em $\alpha\left(t_{i}\right)$ (Figura 6). Assim dado $\epsilon>0$ existe um $\delta>0$ tal que se $|P|<\delta$ então

$$
\left|\int_{a}^{b}\right| \alpha^{\prime}(t)|d t-l(\alpha, P)|<\epsilon .
$$


Pois,

$$
\begin{aligned}
& \lim _{|P| \rightarrow 0} l(\alpha, P)=\lim _{|P| \rightarrow 0} \sum_{i=1}^{n}\left|\alpha\left(t_{i}\right)-\alpha\left(t_{i-1}\right)\right| \\
& \quad \text { Seja } \\
& \left|x\left(t_{i}\right)-x\left(t_{i-1}\right)\right|=\Delta x \text { e }\left|y\left(t_{i}\right)-y\left(t_{i-1}\right)\right|=\Delta y \text { e } P=\left|t_{i}-t_{i-1}\right|=\Delta t
\end{aligned}
$$

Tomando $\Delta t=|P| \forall t \in[a, b]$ temos:

$$
\begin{aligned}
& \lim _{\Delta t \rightarrow 0} \sum \sqrt{\Delta x^{2}+\Delta y^{2}}=\lim _{\Delta t \rightarrow 0} \sum \sqrt{\Delta x^{2}+\Delta y^{2}} \frac{\Delta t}{\Delta t}=\lim _{\Delta t \rightarrow 0} \sum \sqrt{\frac{\Delta x^{2}}{\Delta t^{2}}+\frac{\Delta y^{2}}{\Delta t^{2}}} \Delta t \\
= & \lim _{\Delta t \rightarrow 0} \sum \sqrt{\left[x^{\prime}(t)\right]^{2}+\left[y^{\prime}(t)\right]^{2}} \Delta t=\lim _{\Delta t \rightarrow 0} \sum\left|\alpha^{\prime}(t)\right| \Delta t=\int_{a}^{b}\left|\alpha^{\prime}(t)\right| d t
\end{aligned}
$$

Como $\Delta t=|P|$ e da definição de limite. Dado $\epsilon>0$ existe $\delta>0$ tal que se

$$
|| p|-0|<\delta \Rightarrow\left|l(\alpha, P)-\int_{a}^{b}\right| \alpha^{\prime}(t)|d t|<\epsilon
$$

Assim se

$$
|p|<\delta \Rightarrow\left|\int_{a}^{b}\right| \alpha^{\prime}(t)|d t-l(\alpha, P)|<\epsilon
$$

Logo o comprimento de arco de $\alpha([a, b])$, é o limite de comprimentos de polígonos inscritos.

A aplicação $s(t)=\int_{t_{0}}^{t}\left|\alpha^{\prime}(t)\right| d t$ é denominada função comprimento de arco da curva $\alpha$ a partir do ponto $t_{0}$. Esta função é diferenciável de classe $C^{\infty}$, pois $\alpha$ é uma curva regular.

Definição: uma curva regular $\alpha: I \rightarrow \mathbb{R}^{2}$ é dita parametrizada pelo comprimento de arco, se para cada $t_{0}, t_{1} \in I, t_{0} \leq t_{1} \circ$ comprimento do arco da curva de $t_{0}$ a $t_{1}$ é igual a $t_{1}-t_{0}$, isto é

$$
\int_{t_{0}}^{t_{1}}\left|\alpha^{\prime}(t)\right| d t=t_{1}-t_{0}
$$

\section{Referencial de Frenet}

Como vimos toda curva regular pode ser reparametrizada pelo comprimento de arco. Assim considere a curva regular

$$
\alpha(s)=(x(s), y(s)), s \in I,
$$

parametrizada pelo comprimento de $\operatorname{arcos} s$. Para cada $s \in I, \alpha^{\prime}(s)$ é um vetor unitário, que denotamos por $t(s)$, isto é,

$$
t(s)=\left(x^{\prime}(s), y^{\prime}(s)\right)
$$


Seja $n(s)$ um vetor unitário ortogonal a $t(s)$ tal que a base ortogonal de $\mathbb{R}^{2}$ formada por $t(s)$ e $n(s)$ tem a mesma orientação que a base canônica $e_{1}=(1,0)$ $e_{2}=(0,1)$ de $\mathbb{R}^{2}$, isto é,

$$
n(s)=\left(-y^{\prime}(s), x(s)\right) \text {. }
$$

O conjunto de vetores $t(s)$ e $n(s)$ é dito referencial de Frenet da curva $\alpha$ em $s$. Como o vetor tangente $\alpha^{\prime}(s)$ é unitário, o modulo $\left|\alpha^{\prime \prime}(s)\right|$ da derivada a segunda mede a taxa de variação do ângulo que as tangentes vizinhas fazem com a tangente em $s$. Assim $\left|\alpha^{\prime \prime}(s)\right|$ dá a medida do quão rápido a curva se afasta, em uma vizinhança de $s$, da tangente em $s$ denotada por $|k(s)|$. Temos também que por $\alpha^{\prime}(s)$ ser unitário $\alpha^{\prime \prime}(s)$, é ortogonal a $\alpha^{\prime}(s)=t(s)$, logo $\alpha^{\prime \prime}(s)=t^{\prime}(s)$ é proporcional a $n(s)$. Esse fator de proporcionalidade denotado por $k(s)$, é chamado curvatura de $\alpha$ em $s$, isto é

$$
t^{\prime}(s)=k(s) n(s)
$$

Considerando a curva $\alpha(s)=(x(s), y(s)), s \in I$, segue-se da definição que

$$
k(s)=\left\langle t^{\prime}(s), n(s)\right\rangle=\left\langle\alpha^{\prime \prime}(s), n(s)\right\rangle
$$

Assim

$$
k(s)=-x^{\prime \prime}(s) y^{\prime}(s)+y^{\prime \prime}(s) x^{\prime}(s)
$$

Analogamente, como $n(s)$ é unitário, temos que $n^{\prime}(s)$ é ortogonal a $n(s)$ e, portanto $n^{\prime}(s)$ é proporcional a $t(s)$. Como

$$
\left\langle n^{\prime}(s), t(s)\right\rangle=-x^{\prime}(s) y^{\prime \prime}(s)+x^{\prime \prime}(s) y^{\prime}(s),
$$

concluímos que

$$
n^{\prime}(s)=-k(s) t(s)
$$

Resumindo o exposto acima, se $\alpha: I \rightarrow \mathbb{R}^{2}$ é uma curva regular, parametrizada pelo comprimento de arco $s$, então o referencial de Frenet $t(s), n(s)$ satisfaz as equações

$$
\begin{aligned}
& t^{\prime}(s)=k(s) n(s), \\
& n^{\prime}(s)=-k(s) t(s)
\end{aligned}
$$

que são as formulas de Frenet de uma curva plana.

Teorema fundamental das curvas planas:

a) Dada uma função diferenciável $k(s), s \in I \subset \mathbb{R}$, existe uma curva regular $\alpha(s)$, parametrizada pelo comprimento de arco $s$, cuja curvatura é $k(s)$.

b) A curva $\alpha(s)$ acima é única quando fixamos $\alpha\left(s_{0}\right)=p_{0}$ e $\alpha^{\prime}\left(s_{0}\right)=v_{0}$, onde $v_{0}$ é um vetor unitário de $\mathbb{R}^{2}$. 
c) Se duas curvas $\alpha(s)$ e $\beta(s)$, tem a mesma curvatura, então diferem por suas posição no plano, isto é, existe uma rotação $L$ e uma translação $T$ em $\mathbb{R}^{2}$, tal que

$$
\alpha(s)=(L \circ T)(\beta(s)) .
$$

Demonstração: demonstraremos apenas a parte a) consideremos $\theta(s)=\int_{s_{0}}^{s} k(s) d s$, onde $s_{0} \in I$ é fixo. Fixemos um ponto $p_{0}=\left(x_{0}, y_{0}\right)$ de $\mathbb{R}^{2}$ e $\lambda \in \mathbb{R}$.

Definimos uma curva $\alpha(s)=(x(s), y(s))$ por

$$
\begin{aligned}
& x(s)=x_{0}+\int_{s_{0}}^{s} \cos (\theta(s)+\lambda) d s \\
& y(s)=y_{0}+\int_{s_{0}}^{s} \operatorname{sen}(\theta(s)+\lambda) d s
\end{aligned}
$$

Vamos verificar que a curva $\alpha$ assim definida está parametrizada pelo comprimento de arco $s$ e sua curvatura é $k(s)$. De fato, o referencial de Frenet é

$$
\begin{aligned}
& t(s)=\alpha^{\prime}(s)=(\cos (\theta(s)+\lambda), \operatorname{sen}(\theta(s)+\lambda)), \\
& n(s)=(-\operatorname{sen}(\theta(s)+\lambda), \cos (\theta(s)+\lambda)),
\end{aligned}
$$

e portanto, temos que $\left|\alpha^{\prime}(s)\right|=1$ e a curvatura de $\alpha$ é dada por

$$
\left\langle t^{\prime}(s), n(s)\right\rangle=\theta^{\prime}(s)=k(s) .
$$

\section{DISCUSSÃO}

Temos no caso das curvas planas que o referencial de Frenet se baseia em um diedro ortogonal associado naturalmente a curva plana. Assim para desenvolver a teoria das curvas no espaço é necessário triedro ortogonal onde que junto com os vetores $t(s)$ e $n(s)$, temos o vetor binormal a $\alpha$ em $s$, que é definido por

$$
b(s)=t(s) \times n(s) .
$$

e os vetores $t(s), n(s)$ e $b(s)$ satisfazem as seguintes equações de Frenet

$$
\begin{aligned}
t^{\prime}(s) & =k(s) n(s), \\
n^{\prime}(s) & =-k(s) t(s)-\tau(s) b(s) \\
b^{\prime}(s) & =\tau(s) n(s),
\end{aligned}
$$

Onde $\tau(s)$ é definido por $b^{\prime}(s)=\tau(s) n(s)$ e é denominado torção da curva em $s$. 
Encontro de Ensino, Pesquisa e Extensão, Presidente Prudente, 21 a 24 de outubro, 2013

\section{CONCLUSÃO}

Este trabalho permitiu um contato inicial com os conteúdos essenciais de geometria diferencial, abrindo assim uma oportunidade para uma pesquisa mais aprofundada sobre o assunto

\section{REFERÊNCIAS}

1. TENENBLAT, K. Introdução à geometria diferencial. 2a Ed. São Paulo: Edgard Blucher, 2008. Cap. 1: Curvas planas, p. 28-53.

2. CARMO, M. P. Geometria diferencial das curvas e superfícies. Sociedade Brasileira de Matemática, 2005. Cap. 1: Curvas, p. 2-26. 